\title{
Funciones de la corteza pre frontal en niños de Ayacucho y Lima
}

\section{Pre-frontal cortex functions in children in Ayacucho and Lima}

\author{
ESTHER MARIZA VeLARDe CONSOLI \\ evelardec@unmsm.edu.pe \\ https://orcid.org/0000-0003-0182-4362
}

\author{
Ricardo Celso Canales Gabriel \\ rcanalesg@unmsm.edu.pe \\ https://orcid.org/0000-0003-1195-7281
}

Gonzalo PACHECo LAY

gpachecol@unmsm.edu.pe

https://orcid.org/0000-0002-7888-2301

Universidad Nacional Mayor de San Marcos

\begin{abstract}
RESUMEN:
El presente estudio tuvo como objetivo determinar las diferencias en las funciones de la corteza prefrontal evaluadas a través de las tareas de Clasificación Semántica; Selección de Refranes y Metamemoria entre estudiantes con dos niveles de procedencia geográfica y sociocultural y observar si esos contrastes producían desiguales desempeños. Se realizó de una investigación descriptiva comparativa donde se utilizaron cuatro submuestras de niños de quinto y sexto grado de primaria. Dos de ellas de Ayacucho y dos de Lima. Las cuatro submuestras que fueron seleccionadas son de manera intencional, no probabilística (Sánchez y Reyes, 2006) en instituciones educativas estatales. Como instrumento se utilizó tres subtests de la Batería Neuropsicológica de Funciones Ejecutivas y Lóbulos Frontales de Flores, Ostrosky y Lozano (2012). Los resultados han permitido recomendar acciones preventivas y psicoeducativas para lograr mayores niveles de desempeño en las áreas afectadas por los contextos de pobreza y de falta de estimulación cognitiva y lingüística.
\end{abstract}

\begin{abstract}
:
The present study aimed to determine the differences in the functions of the prefrontal cortex evaluated through the Semantic Classification tasks; Selection of Sayings and Metamemory among students with two levels of geographical and sociocultural origin and observe if these contrasts produced unequal performances. It was a comparative descriptive investigation where four subsamples of children from fifth and sixth grade of primary school were used. Two of them from Ayacucho and two from Lima. The four subsamples that were selected are intentional, not probabilistic (Sánchez and Reyes, 2006) in state educational institutions. As an instrument, three subtests of the Neuropsychological Battery of Executive Functions and Frontal Lobes of Flores, Ostrosky and Lozano (2012) were used. The results have made it possible to recommend preventive and psychoeducational actions to achieve higher levels of performance in the areas affected by the contexts of poverty and lack of cognitive and linguistic stimulation.
\end{abstract}

\section{Palabras Clave:}

Clasificaciones semánticas; selección de refranes; metamemoria; corteza pre frontal anterior.

\section{KEYWORDS:}

Semantic classifications; saying selection; metamemory; previous pre-frontal bark.

Recibido: 05/03/2021 - Aceptado: 11/03/2021 - Publicado: 23/06/2021

\footnotetext{
(C) Los autores. Este artículo es publicado por la Revista Peruana de Investigación e Innovación Educativa de la Facultad de Educación, Universidad Nacional Mayor de San Marcos. Este es un artículo de acceso abierto, distribuido bajo los términos de la licencia Creative Commons Atribución 4.0 Internacional (CC BY 4.0) [https://creativecommons.org/licenses/by/4.0/deed.es] que permite el uso, distribución y reproducción en cualquier medio, siempre que la obra original sea debidamente citada de su fuente original.
} 


\section{Introducción}

Actualmente, se ha dado mucha importancia a las funciones cognitivas que expresan el nivel de funcionamiento de los lóbulos frontales que son las áreas de más reciente evolución del cerebro (Goldberg, 2001). Una de ellas, es la corteza prefrontal cuya porción, más anterior, presenta una organización estructural exclusiva sólo de la especie humana (Stuss y Levine, 2002). Su nivel de funcionamiento son las medidas con tareas de clasificación semántica; la selección de refranes y la metamemoria que consiste en la habilidad metacognitiva para desarrollar estrategias de almacenamiento y realizar juicios de predicción de desempeño.

Las funciones lingüísticas y la metamemoria, evaluadas en la presente investigación, son ejercidas por las áreas de la corteza frontal. Estas, como ya se ha mencionado, son aquellas de más reciente evolución humana (Luria, 1989). Sus funciones son fundamentales, puesto que cumplen tareas que nos permiten enfrentar satisfactoriamente a las demandas del mundo moderno. Una de ellas está relacionada con la función de planeación que nos permite ordenar y secuenciar un conjunto de procesos hasta alcanzar una meta trazada. Esto es posible, gracias a la función de abstracción que nos posibilita: percibir, analizar y secuenciar el conjunto de actividades físicas y mentales que realizamos con el fin de lograr asimilar de manera más coordinada, la información que ingresa a nuestro sistema mental que requiere ser procesada.

También, cumple la función de organizar los estímulos llegando a clasificarlos en categorías semánticas y regular nuestra conducta permitiéndonos planear y controlar nuestro comportamiento ejerciendo un control inhibitorio sobre nuestras respuestas impulsivas, manejando nuestras emociones adecuadamente e insertándonos en el mundo social de manera independiente, asertiva y proactiva (Stuss y Levine, 2002). Por todo ello, algunos autores (Goldberg, 2001) la denominan como el centro ejecutivo del cerebro.

Otra función que cumple es aquella que nos permite actuar con flexibilidad mental, creando nuevas estrategias y repuestas ante situaciones donde se ha variado el contexto situacional. Además, la memoria de trabajo es aquella que logra que el sujeto pueda realizar tareas simultáneas manteniendo presente la información requerida mientras se procesa la siguiente. Por último, la capacidad de realizar tareas metacognitivas que nos permite ejercer un control sobre nuestro desempeño con el fin de monitorearlo, regularlo y mejorarlo para aportar de mejor manera a la sociedad.

Los lóbulos frontales se sitúan encima de la cisura lateral y delante de la cisura central. Se dividen en tres grandes regiones: dorsolateral, orbital y medial y se conforman gracias a un proceso madurativo. Este proceso evoluciona hasta que el sujeto alcanza la edad adulta (Lenroot y Gied, 2006) que depende de dos acciones, la progresiva que está relacionada con la proliferación celular. Una de ellas es la denominada: la arborización dendrítica, que tiene que ver específicamente con el nacimiento y el crecimiento de ellas y la otra, conocida como la mielinización, que es la actividad relacionada con el recubrimientos de los axones de la neuronas con mielina cuyo proceso se completa en la tercera década de vida, y las regresivas donde se producen la apoptosis (muerte celular programada) y la poda neuronal (eliminación de conexiones cerebrales) que continúa hasta los 16 años (Capilla et al., 2004). Pero, los procesos biológicos de maduración evolucionan junto con las experiencias de aprendizaje, determinado por factores socioculturales, cuya calidad y cantidad de estas garantizarán un buen nivel de desempeño de las funciones ejecutivas (Hackman y Farah, 2008).

La región orbitofrontal, es la zona que se encuentra estrechamente vinculada con el sistema límbico, por lo que su función principal, es la regulación y el procesamiento de las emociones y los estados afectivos (Damasio, 1998). Asimismo, se encarga de evaluar las condiciones de riesgo o de beneficio situacionales, lo que le permite al sujeto, a partir de toma de decisiones, realizar los suficientes ajustes a los patrones de comportamiento (Bechara, Damasio y Damasio, 1996).

La región medial se encarga de las tareas atencionales y motivacionales relacionadas con la detección de conflictos cuya solución requieren activar los procesos de regulación e inhibición de los impulsos agresivos (Bagaiyan y Posner, 1997). Las porciones más anteriores de la corteza medial se encuentran involucradas 
en la mentalización o la denominada teoría de la mente que es concebida como la capacidad de atribuir pensamientos e intenciones a otras personas (Shallice, 2001).

La región dorsolateral está encargada de los procesos ejecutivos de planeación, la memoria de trabajo, las tareas de fluidez y flexibilidad mental, la seriación y secuenciación de las estrategias de trabajo, generación de hipótesis y la solución de problemas complejos (Stuss y Alexander, 2000). Sus porciones realizan actividades de jerarquía cognitiva como la metacognición (monitoreo y ajuste) y la integración de las experiencias emocionales (Stuss y Levine, 2002).

Para finalizar, es importante reiterar que muchos autores (Morris, Ahmed, Syed, y Toone, 1993) señalan que existen diferencias entre el nivel de funcionamiento de la corteza frontal izquierda frente a la derecha. Donde la corteza frontal izquierda se relaciona más con los procesos de la flexibilidad mental, la planeación secuencial, la tarea de secuencias inversas, la fluidez verbal y la memoria de trabajo. Mientras que, la derecha se relaciona más con la construcción y el diseńo de objetos y figuras, la memoria de trabajo para material visual, la apreciación del humor y la memoria episódica.

Los objetivos del presente trabajo fueron investigar si existían diferencias en las tareas relacionadas con el nivel de desarrollo de las funciones neuropsicológicas ejecutivas del área prefrontal en niños, con distinto lugar de procedencia geográfica y sociocultural. En este caso, se tomaron dos realidades que fueron Ayacucho y Lima para comprobar, si existían diferencias en la tarea de clasificación semántica, en la tarea de selección de refranes y en metamemoria; es decir, en términos globales, en el nivel de las funciones neuropsicológicas de la corteza prefrontal anterior, en niños de distinto lugar de procedencia geográfica y sociocultural.

Este estudio se enmarca en la investigación sobre los aportes y avances de las neurociencias cognitivas actuales, interesadas actualmente, en temas de diagnóstico de las áreas neuropsicológicas. En tal sentido, apuntan a entender la relación entre la educación y las neurociencias, pues nos permite encontrar más información sobre el estado del funcionamiento cerebral y las repercusiones sobre el aprendizaje escolar. Asimismo, sienta un precedente importante en la investigación neuropsicológica en educación.

Como antecedentes tenemos los estudios realizados por Gómez, Rubinstein y Politis (2014) quienes investigaron las repercusiones de la memoria de trabajo sobre el rendimiento práxico. Evaluaron el funcionamiento de la memoria de trabajo (gestual, visual y verbal) sobre el rendimiento práxico en 23 pacientes con demencia fronto-temporal. Encontrando diferencias entre el rendimiento en memoria de trabajo gestual sobre la producción de praxias, concluyendo la posibilidad de una memoria de trabajo específica de gestos.

Por su parte, Reyes, Montes, González y Pinto (2013) evaluaron la clasificación de roles semánticos basada en características sintácticas, semánticas y contextuales. El objetivo fue identificar mediante la tarea de clasificación, el tipo de rol semántico existente entre un evento y sus actantes. Encontrando que la selección de atributos mejoraba el desempeńo de la tarea de clasificación. Concluyeron que el resultado del etiquetado de roles puede ser utilizado para una representación de conocimiento.

Asimismo, Aguirre de Ramírez (2000) estudió las estrategias usadas por un grupo de estudiantes de quinto grado de primaria para entender y analizar refranes populares tanto a nivel oral como escrito. Encontró que la forma de razonar de los niños demostraba que trabajan guiados por el sentido figurado dándose cuenta de que las expresiones que debían analizar estaban constituidas por palabras diferentes que podían significar lo mismo. Tres de los niños abordaron la tarea mecánicamente. El resto desarrolló argumentos de interpretación que se le da socialmente en función de su uso.

Este estudio permitió determinar si las condiciones de marginalidad económica, productiva y sociocultural influyeron en las funciones cognitivas y de metamemoria que expresan el nivel de desempeño del área prefrontal anterior del cerebro para, a partir de estos hallazgos, recomendar programas preventivos o de rehabilitación de las áreas afectadas. 


\section{Método}

El presente estudio, es del tipo investigación sustantiva descriptiva, pues estuvo orientada a identificar los niveles de desempeño en tareas de clasificación semántica, selección de refranes y de metamemoria en estudiantes con distinto lugar de procedencia sociocultural pertenecientes a instituciones educativas estatales de Lima y Ayacucho. El diseño empleado fue el descriptivo comparativo debido a que se pretendió hallar diferencias en los rendimientos de las variables seleccionadas que expresan el nivel de funcionamiento de la corteza pre frontal anterior entre las cuatro submuestras planteadas. El método fue cuantitativo y cualitativo, pues las variables fueron valoradas numéricamente y también interpretadas cualitativamente como aquellos aspectos relacionados a los niveles de abstracción y de metamemoria evaluadas.

Se utilizaron tres subtests de la Batería Neuropsicológica de Funciones Ejecutivas y Lóbulos Frontales BANFE-2 de Flores, Ostrosky y Lozano (2012). Este instrumento neuropsicológico está diseñado para evaluar el desempeño de las funciones ejecutivas en sujetos de habla hispana comprendidos desde los seis años hasta la edad adulta, a través de 15 procesos vinculados con las funciones ejecutivas agrupadas en tres áreas cerebrales específicas: dorsolateral, prefrontal anterior y orbito medial.

Es una batería de pruebas que evalúa las distintas áreas anátomo-funcionales de los lóbulos frontales. Por ejemplo, las pruebas como Efecto Stroop (que evalúa la capacidad de control inhibitorio); Juego de cartas (capacidad para evitar selecciones de riesgo y beneficio) y Laberintos (capacidad de seguir reglas y respetar límites) evalúan el nivel de funcionamiento de la corteza orbitofrontal (COF) y la corteza prefrontal medial (CPEM).

Por otro lado, las pruebas que evalúan la calidad en el funcionamiento de la Corteza PreFrontal Dorso Lateral (CPDL) son: señalamiento autodirigido (memorizar información visoespacial de forma autodirigida); la memoria de trabajo visoespacial (retener una secuencia visoespacial); ordenamiento alfabético de palabras (ordenar mentalmente la información verbal); clasificación de cartas (cambiar el criterio de clasificación); laberintos (planear la conducta visoespacial); Torres de Hanoi (anticipar acciones en orden progresivo y regresivo); suma y resta consecutiva (secuenciar en orden inverso) y fluidez verbal (producir de forma fluida la mayor cantidad de verbos). Finalmente, las pruebas que evalúan funciones que dependen principalmente de la Corteza Prefrontal Anterior (CPFA) son: la Selección de refranes, las Clasificaciones semánticas y la Metamemoria.

La Selección de refranes es una prueba que fue propuesta por Luria, en el año 1989, para evaluar a los pacientes con daño frontal con el objetivo de comparar su rendimiento frente a otros con lesiones en otras áreas del cerebro. En este subtest el sujeto requiere realizar una tarea abstracta de procesamiento semántico y sintáctico de las palabras que componen un refrán para acceder al significado bi - textual (González, 2006) a través de un ejercicio lingüístico y cognitivo que requiere un análisis de tipo inferencial (Nippold y Haq, 1996). Es decir, una tarea cognitiva que exige identificar el mensaje implícito del contenido verbal.

Es necesario mencionar la prueba anteriormente mencionada está conformada por cinco refranes (sugeridos por Lezak, 1994; Nippol y Haq, 1996; Barth y Kufferie, 2001) donde el examinado deberá leer en voz alta y luego seleccionar de tres alternativas presentadas aquella que encierre el mensaje esencial del refrán leído. El examinado debe seleccionar una de los tres en un tiempo máximo de cinco minutos. Como es una tarea compleja se aplica a infantes a partir de los 10 ańos. Tiene mayor puntuación las respuestas correctas.

Las Clasificaciones Semánticas fueron propuestas por Delis, Squire, Birle y Massman (1992). Es un subtest que mide la capacidad de abstracción y flexibilidad mental a través de la actividad de realizar categorías semánticas con distintos criterios. Según Reber, Stark y Squire (1998); Bright, Moss y Tyler (2014) y Nopponey, Price, Penny y Friston (2005) el realizar este tipo de tareas genera la activación del área 10 de la Corteza Prefrontal anterior, en especial, la izquierda. Al evaluado se le muestra una lámina que contiene 30 figuras de animales y se le pide que agrupe las figuras utilizando algún criterio de clasificación que deberá 
verbalizar. El examinado dispone de cinco minutos como tiempo máximo para realizar esta tarea. Obtiene más alta puntuación aquellos sujetos que logran realizar mayor número de clasificaciones y de mejor nivel de abstracción. El sujeto puede realizar distintos tipos de clasificaciones.

Con relación a las clasificaciones, la primera de ellas denominada concretas y es cuando agrupa considerando las características físicas de los animales. Por ejemplo, el niño puede decir: agrupé a los animales que tienen cuatro patas. Lo segundo, son las categorías funcionales cuando clasifica considerando algunas actividades de los animales. Por ejemplo, dice: agrupé a todos los animales peligrosos o los animales que viven en el agua. Un tercer nivel de categoría es la abstracta que se expresa cuando agrupa con criterios más elaborados como, por ejemplo: porque son mamiferos, porque son domésticos, entre otros. Las categorías concretas valen un punto, las funcionales dos y las abstractas tres. Además, se consideran el número de elementos que incluye en cada categoría.

La metamemoria también fue una prueba propuesta por Luria en el año 1989 y forma parte de otro instrumento denominado Batería de metamemoria de Belmont y Borkowski (1988). Es denominada metamemoria porque exige un monitoreo de la memoria y un control metacognitivo. Esta prueba exige la activación de las áreas prefrontales anteriores según revelan las pruebas de neuroimagen funcional (FernándezDuque, Baird y Posner, 2000; Shimamura, 2000). Este subtest evalúa la capacidad del sujeto de realizar predicciones sobre sus propios juicios de desempeño memorístico.

La actividad consiste en aprenderse nueve palabras que son presentadas verbalmente por el examinador durante cinco ensayos y luego se le pregunta al examinado, antes de cada resultado, cuántas cree que podrá memorizar; es decir, el sujeto deberá realizar una predicción sobre su propia capacidad de almacenamiento. Esta actividad también permitirá registrar una situación llamada intrusión (cuando expresa palabras que no estaban en la lista) o perseveración (cuando repite la misma palabra de la lista varias veces). Tendrá un desempeño de sobreestimación cuando predice un mayor número de palabras de las que finalmente recordó o de subestimación cuando predice un menor número de palabras de lo que finalmente logró. Para la puntuación se tendrá en cuenta el número de palabras recordadas en cada ensayo y la resta entre el número de las palabras predichas en cada ensayo con el total de palabras recordadas.

Con respecto a la confiabilidad y validez del instrumento, los autores de la prueba sostienen que consideraron el criterio de validez clínica, convergente y de constructo (Stuss y Levine, 2002), puesto que el conjunto de instrumentos componentes de la batería han sido utilizados ampliamente por la comunidad neuropsicológica en pacientes con y sin daño cerebral y cuyos desempeños han sido corroborados con las pruebas de neuroimagen funcional, obteniendo un nivel alto de confiabilidad de 0.80 . Los autores, además precisan que obtuvieron las normas seleccionando a 450 sujetos de entre 6 y 85 ańos de edad, sin trastornos ni antecedentes de ningún tipo de dificultad neuropsicológica. A partir de los puntajes se clasifican los sujetos como: normal alto; normal, alteraciones leves a moderadas y alteraciones severas. También, ofrece un perfil en cada una de las áreas neuropsicológicas evaluadas; sin embargo, en nuestra investigación no utilizamos las normas de clasificación propuesta sino realizamos interpretaciones de carácter cualitativo.

El sustento metodológico de la prueba se basa en un criterio de análisis cuantitativo y cualitativo puesto que, de acuerdo al modelo sistémico de Luria, existe un conjunto de áreas que ejercen un papel específico dentro del sistema y que actúan de manera interactiva entre ellas de tal manera que se podría detectar el área cortical afectada.

La muestra estuvo compuesta por todos 80 alumnos de quinto y sexto grado que estudiaban en la institución educativa Guamán Poma de Ayala y Gustavo Castro Pantoja de Ayacucho y 80 estudiantes de los colegios $N^{\circ} 0014$ Andrés Bello y la institución educativa $N^{\circ} 1076$ Rabindranath Tagore de la ciudad de Lima. Estos datos han sido expresados en la tabla 1.

Se utilizaron tres subtests de la Batería Neuropsicológica de Funciones Ejecutivas y Lóbulos Frontales de Flores, Ostrosky y Lozano (2014). Los subtests de Clasificación Semántica, Selección de Refranes y Metamemoria. 
Tabla 1

Distribución de los Participantes por Lugar de Procedencia

\begin{tabular}{cccc}
\hline Región & Colegio & F & $\%$ \\
\hline Ayacucho & Guamán Poma de Ayala & 40 & 25 \\
Ayacucho & Gustavo Castro Pantoja & 40 & 25 \\
Lima & Andrés Bello & 40 & 25 \\
Lima & Rabindranath Tagore & 40 & 25 \\
\hline
\end{tabular}

\section{Resultados y discusión}

En la tabla 2, se aprecian los resultados obtenidos tras realizar las comparaciones en las tareas de Clasificación Semántica, considerando el número de categorías abstractas (S2-11). La clasificación semántica, como ya se ha afirmado, es una tarea que evalúa dos aspectos, en primer lugar, el nivel de abstracción que posee el sujeto manifestado por la generación de diversas categorías semánticas que emite en un tiempo indicado (cinco minutos) y, en el segundo lugar, por el grado de productividad léxica al ser capaz de emitir el mayor número de elementos pertenecientes a la categoría elegida.

Es necesario mencionar que son registradas las respuestas que consideran aspectos concretos de los animales, como el número de patas o el tamaño de los animales, o las que expresan un criterio funcional como cuando los selecciona, considerando algunas capacidades de los animales; como decir, que son peligrosos o que son rápidos. Pero es desde los 10 años que se pide que los niños sean capaces de clasificar a los animales considerando las características abstractas de ellos, como si son mamíferos o marinos. La puntuación máxima es de 36.

También se aprecian los resultados obtenidos tras realizar las comparaciones en las tareas de Clasificación Semántica, considerando el número de categorías abstractas (S2-11); en el tiempo empleado en la Selección de Refranes (S2-12); en los aciertos obtenidos en la Selección de Refranes (S2-13); en los errores negativos en la Metamemoria (S2-14) y en los errores positivos en la Metamemoria (S2-15). En el caso de este último subtest, el tener un mayor número de errores implica una incapacidad de monitorear su propio desempeńo, actividad relacionada con una de las funciones de la corteza prefrontal anterior, puesto que, ante cada una de las opciones que emite el sujeto, el examinador le va informando el resultado obtenido y se supone que el control metacognitivo de los lóbulos frontales permitirá que el sujeto vaya ajustando sus respuestas.

Se compararon todas las tareas correspondientes al funcionamiento neuropsicológico del área Pre Frontal Anterior de la Corteza Cerebral entre estudiantes de Ayacucho y Lima, encontrándose que solo existen pequeñas diferencias estadísticamente significativas en el S2-11: Clasificación Semántica, a favor de los estudiantes de Ayacucho. También, se observa a un nivel práctico, que existen pequeñas diferencias en el S212: Selección de Refranes en este caso, también a favor de los estudiantes de Ayacucho.

Tabla 2

Diferencias en las Tareas Vinculadas con el Área del Córtex Prefrontral Según el Lugar de Procedencia

\begin{tabular}{|c|c|c|c|c|c|c|c|}
\hline \multirow{2}{*}{$\begin{array}{l}\text { Tareas área } \\
\text { pre frontal }\end{array}$} & \multicolumn{2}{|c|}{$\begin{array}{c}\text { Ayacucho } \\
(n=80)\end{array}$} & \multicolumn{2}{|c|}{$\begin{array}{c}\text { Lima } \\
(n=80)\end{array}$} & \multirow[t]{2}{*}{$\mathbf{t}$} & \multirow[t]{2}{*}{$\mathbf{p}$} & \multirow[t]{2}{*}{ d } \\
\hline & $M$ & $\mathrm{DE}$ & $M$ & $\mathrm{DE}$ & & & \\
\hline S2-11 & 4.1 & (1.93) & 3.4 & (2.11) & 2.192 & $.030^{*}$ & .35 \\
\hline S2-12 & 134.66 & $(70.11)$ & 154.88 & (79.16) & -1.711 & .089 & .27 \\
\hline S2-13 & 3.44 & $(0.87)$ & 3.31 & (0.89) & .987 & .325 & .15 \\
\hline S2-14 & 3.88 & (2.63) & 3.7 & $(2.86)$ & .402 & .688 & .07 \\
\hline S2-15 & 3.23 & (2.52) & 3.04 & (2.61) & .462 & .645 & .07 \\
\hline
\end{tabular}




\section{Discusión}

El presente estudio tuvo como fin observar si existían diferencias en las tareas de Clasificación Semántica, Selección de Refranes y Metamemoria, que son tareas cognitivas relacionadas con el nivel de funcionamiento de la Corteza Pre Frontal Anterior, entre estudiantes de Ayacucho y Lima. Sólo se encontraron leves diferencias en la tarea de Clasificación Semántica y favor de los estudiantes de Ayacucho. El primer colegio que se evaluó estuvo conformado por los alumnos de la escuela de aplicación de la Universidad San Cristóbal de Huamanga de Ayacucho, donde en su gran mayoría estudian los hijos de los profesores de dicha universidad. Se trató de una muestra de 40 alumnos entre 10 a 12 años que cursaban el quinto y sexto grado de primaria en el colegio de aplicación: Guamán Poma de Ayala. La gran mayoría de sus padres y madres presentaron instrucción superior y ejercían actividades profesionales como ingenieros, abogados y profesores. Eran bilingües y contaban, en sus viviendas, con servicios básicos de agua, luz y desagüe. De esta submuestra un alto número de alumnos logró realizar hasta 5 clasificaciones abstractas ante la tarea demandada.

Con relación a la segunda submuestra de Ayacucho esta estuvo conformada también por 40 estudiantes, entre 10 a 13 años, de la Institución Educativa Estatal Gustavo Castro Pantoja. A diferencia de la primera, existió un grupo considerable de padres y madres que tenían solo educación primaria y se dedicaban a profesiones de menor exigencia académica como la actividad de constructor o albañil. En el caso de esta submuestra hubo un menor número de estudiantes que lograron más de 4 categorías abstractas. Para el presente estudio se realizó una sumatoria de ambos grupos.

En el caso de los grupos de Lima, hubo 40 estudiantes entre 10 y 12 años pertenecientes a la Institución Educativa Andrés Bello del distrito de Pueblo Libre, en Lima. En este grupo, todos los padres habían estudiado hasta educación secundaria, más no ocurría lo mismo en el caso de las madres. Además, dentro de la muestra hubo un reducido grupo con educación superior. En cuanto a las tareas profesionales, se encontró un mayor número de madres que sólo eran amas de casa. En la prueba de clasificación semántica, hubo un menor número de alumnos que lograron hasta cinco agrupaciones abstractas y, en términos generales, el desempeño fue mejor que el Gustavo Castro Pantoja de Ayacucho. En cuanto al colegio Rabindranath Tagore del distrito de San Miguel, en Lima, se encontró un reducido grupo de padres y madres con educación primaria pero el número de estudiantes que lograron cinco categorías abstractas fue similar al de Gustavo Castro Pantoja de Ayacucho.

Realizando un análisis cualitativo, se observó que el grado de instrucción y la profesión de los padres era el factor que más incidía sobre la capacidad de establecer categorías abstractas. Es por ello, por lo que los estudiantes de la institución educativa Guamán Poma de Ayala de Ayacucho quienes obtuvieron mayores desempeños que los dos colegios de Lima. Aquí se ve como el lugar de procedencia geográfica no es un factor que, necesariamente, repercute en las distancias en los desempeños, puesto que los alumnos de Ayacucho rindieron mejor que los de Lima. Fue, más bien, el factor educativo de los padres, el que marcó la diferencia y, se añade también, la capacidad de gestión educativa. En el caso del colegio de aplicación de Ayacucho, se evidenciaba un alto nivel de organización de sus actividades y una minuciosa supervisión de la calidad educativa de sus actividades académicas.

La siguiente tarea fue la Selección de Refranes cuyo objetivo fue interpretar bi-textualmente el significado de cinco refranes. En el primer caso, se controla el grado de velocidad empleado y, paradójicamente, se observa que los estudiantes pertenecientes a las instituciones educativas de Ayacucho, en especial los de Guamán Poma de Ayala, lo hicieron en menor tiempo, aunque no se encontraron diferencias significativas entre ambas muestras. En cuanto a los aciertos obtenidos en la Selección de Refranes, también dichos estudiantes lograron un mejor desempeño en la habilidad de encontrar el lenguaje bi-textual contenido en los refranes; sin embargo, tampoco se observaron diferencias significativas comparando ambas regiones. En cuanto a la Metamemoria, fueron los alumnos de Lima, los que lograron un mejor desempeńo en esta actividad, aunque las diferencias tampoco fueron significativas. 
Para dar respuesta al objetivo central, que fue determinar si existían diferencias en las tareas relacionadas con el nivel de funcionamiento del Área Pre Frontal anterior en niños de distinto lugar de procedencia sociocultural, concluimos que sólo se encontraron diferencias significativas en las tareas de Clasificación Semántica y a favor de los estudiantes de Ayacucho; en especial, en aquellos que estudiaban en el colegio de aplicación: Guamán Poma de Ayala, cuyas diferencias con los otros grupos, fue que los padres de esos alumnos tenían mayor nivel de instrucción y profesión.

Es posible afirmar, que el lugar de procedencia (capital versus región) no determina un menor nivel de desempeño, sino que es la calidad de la formación del estudiante, que es promovida por el nivel de instrucción y profesión de los padres aquello que marca las diferencias. Asimismo, el grado de organización y calidad en la gestión educativa, por lo menos en lo relacionado al pensamiento categorial abstracto.

También, se observaron mejores desempeños a favor de los estudiantes de Ayacucho, en especial al del colegio de aplicación, en las tareas relacionadas con la rapidez en la selección de refranes interpretando el significado bi-textual de los mismos, mas no tanto en la selección adecuada de las respuestas. Asimismo, se observaron diferencias no significativas en las tareas de meta control del desempeño de la memoria y el control cognitivo de esta actividad a favor de los estudiantes de Lima; sin embargo, haciendo una aproximación neuropsicológica del desempeńo de los grupos se observa un mayor porcentaje de estudiantes de la institución Guamán Poma de Ayala con un performance más adecuada, quedando en segundo lugar los del colegio Andrés Bello de Lima, así como un tercer y cuarto lugar para las otras dos instituciones. Un mayor número de alumnos con déficits marcados se encontraron en el colegio Gustavo Castro de Ayacucho seguido por los dos de Lima, siendo la institución Guamán Poma de Ayala de Ayacucho donde se encontró solo un caso con este tipo de dificultades. Estos datos son indicadores que revelan la necesidad de una evaluación más exhaustiva.

\section{Conclusiones}

A partir el estudio realizado se puede evidenciar que se encontraron diferencias significativas en la tarea de Clasificación Semántica (S2-11) a favor de los estudiantes de Ayacucho. También es preciso señalar que no se encontraron diferencias significativas en la velocidad empleada en la tarea de Selección de Refranes (S212). Asimismo, no se encontraron diferencias significativas en la tarea de Selección de Refranes (S2-13). Por otro lado, no se encontraron diferencias significativas en los errores positivos de la Metamemoria (S2-14). Finalmente se puede asumir que no se encontraron diferencias significativas en los errores negativos de la Metamemoria (S2-15).

\section{Nota de reconocimiento}

Queremos agradecer a los estudiantes de la Universidad Nacional Mayor de San Marcos que demostraron compromiso con la investigación, la universidad y el país acompańándonos en los lugares seńalados para el estudio y desempeñando eficiencia en las coordinaciones, evaluaciones y registro de los datos. De la Facultad de Educación: ANAIS PABLO VILLANUEVA, YOLY RODRÍGUEZ MELENDEZ, VANESSA JURADO MANDUJANO, ANTHONY TRUJILLO MASFIL, DULCE IGLESIAS LANDA, KIARA ESTUPIÑAN GONZALES, LIZ GAVILAN CAARHUPOMA y MIRIAM SOLIS HUAQUISACA y de la Facultad de Psicología: JUAN RAMIREZ MENDOZA, CARMEN ANGELIKA PIZARRO NEGRÓN, HELEN SILVA GUTIERREZ, DANIELA CASTAÑEDA PEREZ, JEANPEER VILA VENTOCILLA, FLOR BASTIDAS ALVARADO, SUSY FLORES GUERRA, TATIANA CANCHES HERNANDEZ, y LEANDRA CCOYLLO GONZALEZ. Así como a los Directores, personal educativo y administrativo, niños y padres de familia que nos dieron todas las facilidades para la evaluación: la Institución Educativa 1076 RABINDRANATH TAGORE del distrito de Pueblo Libre, en la ciudad de Lima; la Institución Educativa 0014 ANDRÉS BELLO del distrito de Pueblo Libre, en la ciudad de Lima; la Institución Educativa COLEGIO HUAMAN POMA DE AYALA de la ciudad de Huamanga, en Ayacucho, y la Institución Educativa 38001 GUSTAVO CASTRO PANTOJA, también de la ciudad de Huamanga, en Ayacucho, A todos ellos un sincero agradecimiento por su contribución al estudio y a la investigación en nuestro país. 


\section{Referencias}

Aguirre de Ramírez, R. (2000). La comprensión del sentido de los refranes en escolares. Educere, 3(9), 76-83. https:// www.redalyc.org/pdf/356/35630913.pdf

Bagaiyan, R., y Posner, M. (1997). Time course activations in implicit and explicit recall. Journal of neuroscience, 17(12), 4904-4913. https://www.nature.com/articles/nrn1201

Barth, A., y Kufferie, B. (2001). Development of a proverb test for assessment of concrete thinking problems in schizophrenic patients. En: Flores, J.C. y Ostrosky-Shejet, F. y Lozano, A. (2012). BANFE. Batería neuropsicológica de funciones ejecutivas y lóbulos frontales. Manual Moderno.

Bechara, A. Damasio, H., y Damasio, A. (1996). Failure to respond autonomically to anticiped future outcomes following damage to pre frontal cortex. Cerebral cortex. 6(2), 215-225. https://pubmed.ncbi.nlm.nih.gov/8670652/

Belmont, J., y Borkowski, J. (1988). A group test of children's matamory. En: Flores, J.C. y Ostrosky-Shejet, F. y Lozano, A. (2012). BANFE. Batería neuropsicológica de funciones ejecutivas y lóbulos frontales. Manual Moderno.

Bright, P., Moss, H., y Tyler, L. (2014). Unitary vs multiple semantics, PET studies of Word and picture processing. Brain and Laguage. 89. En: Flores, J.C. y Ostrosky-Shejet, F. y Lozano, A. (2012). BANFE. Batería neuropsicológica de funciones ejecutivas y lóbulos frontales. Manual Moderno.

Capilla, A., Romero, D., Maestú, F., Campo, P. Fernández, S., y Ortiz, T. (2004). Emergencia y desarrollo cerebral de las funciones ejecutivas. Actas españolas de psiquiatría, 32(6), 377-386. https://medes.com/publication/15756

Damasio, A. (1998). The somatic marker hypothesis and the possible functions of the pre frontal cortex. En: Flores, J. y Ostrosky, F. (2012). Desarrollo neuropsicológico de lóbulos frontales y funciones ejecutivas. Manual Moderno.

Delis, D., Squire, L., Birle, A., y Massman, P. (1992). Componential analysis of problem solving ability: performance of patients with frontal lobe damage and amnesic patients on a new sorting test. En: Flores, J.C. y OstroskyShejet, F. y Lozano, A. (2012). BANFE. Batería neuropsicológica de funciones ejecutivas y lóbulos frontales. Manual Moderno.

Fernández-Duque, D., Baird, J., y Posner, M. (2000). Executive attention and metacognitive regulation. Consciousness and cognition. 9. En: Flores, J.C. y Ostrosky-Shejet, F. y Lozano, A. (2012). BANFE. Batería neuropsicológica de funciones ejecutivas y lóbulos frontales. Manual Moderno.

Flores, J.C., Ostrosky-Shejet, F., y Lozano, A. (2012). BANFE. Batería neuropsicológica de funciones ejecutivas y lóbulos frontales. Manual Moderno.

Goldberg, E. (2001). The executive brain, frontal lobes and the civilized mind. Oxford University Press. En: Flores, J. y Ostrosky, F. (2012). Desarrollo neuropsicológico de lóbulos frontales y funciones ejecutivas. Manual Moderno.

Gómez, P., Rubinstein, W., y Politis, D. (2014). Déficit en producción motora y severidad de la demencia en Alzheimer y demencia frontotemporal. Revista Neuropsicología Latinoamericana, 6(1),1-12. http://pepsic.bvsalud.org/scielo. php?script=sci_arttext\&pid=S2075-94792014000100001

González, R. (2006). Problemas psicolingüisticos en el Perú. Obras Completas. Volumen I. Ediciones Norma Reátegui.

Hackman, D., y Farah, M. (2008). Socioeconomic status and developing brain. Trends and cognitive Sciences, 13(2). https://pubmed.ncbi.nlm.nih.gov/19135405/

Lenroot, R., y Gied, J. (2006). Brain development in children and adolescents: insights from anatomical magnetic resonance imaging. Neuroscience and Biobehavioral Review, 30(6), 718-29. https://pubmed.ncbi.nlm.nih. gov/16887188/

Lezak, M. (1994). Neuropyshcological Evaluation. Oxford University Press.

Luria, A.R. (1989). Las funciones corticales superiores del hombre. México: Fontamara.

Morris, R., Ahmed, S., Syed, M., y Toone, B. (1993). Neural correlates of planning ability: frontal lobe activation during the tower of London test. Neurosychologia, 31 (2). https://www.sciencedirect.com/science/article/abs/ pii/0028393293901048 
Nippold, M., y Haq, F. (1996). Proverb compression in youth: the role of concreteness and familiarity. Journal of Speech and Hearing research, 39(1), 166-76. https://pubmed.ncbi.nlm.nih.gov/8820708/

Nopponey, U., Price, C. Penny, W., y Friston, K. (2005). Two distinct neural mechanisms for category selective responses. Cerebral córtex. 16. En: Flores, J.C. y Ostrosky-Shejet, F. y Lozano, A. (2012). BANFE. Batería neuropsicológica de funciones ejecutivas y lóbulos frontales. Manual Moderno.

Reber, P., Stark, C., y Squire, L. (1998). Cortical areas supporting category learning identified using fMRI. Proceding of the National Academt of Sciences USA. En: Flores, J.C. y Ostrosky-Shejet, F. y Lozano, A. (2012). BANFE. Batería neuropsicológica de funciones ejecutivas y lóbulos frontales. Manual Moderno.

Reyes, J., Montes A., González, J., y Pinto D., (2013). Clasificación de roles semánticos usando características sintácticas, semánticas y contextuales. Computación y Sistemas, 17(2), 263-272. http://www.scielo.org.mx/scielo. php?script=sci_arttext\&pid=S1405-55462013000200016

Sánchez, H., y Reyes, C. (2006). Metodología y diseños en la investigación científica. Editorial Universidad Ricardo Palma.

Shallice, T. (2001). Theory and mind and the pre frontal cortex. Brain, 124(2). https://academic.oup.com/brain/ article/124/2/247/402213?login=true

Shimamura, A. (2000). Toward and cognitive neuroscience of metacognition. Consciousness and cognition. En: Flores, J.C. y Ostrosky-Shejet, F. y Lozano, A. https://www.sciencedirect.com/science/article/abs/pii/S1053810000904501

Stuss, D., y Levine, B. (2002). Adult clinic neuropsychology: lessons from studies of the frontal lobes. En: Flores, J. y Ostrosky, F. (2012). Desarrollo neuropsicológico de lóbulos frontales y funciones ejecutivas. BANFE. Batería neuropsicológica de funciones ejecutivas y lóbulos frontales. Manual Moderno. México.

Stuss, D., y Alexander, M. (2000). Executive functions and the frontal lobes: a conceptual view. Psychology Research, 63(3), 289-98. https://pubmed.ncbi.nlm.nih.gov/11004882/

\section{Como citar este artículo:}

Velarde, E., Canales, R. y Pacheco, G. (2021). Funciones de la corteza pre frontal en nińos de Ayacucho y Lima. Revista Peruana de Investigación e Innovación Educativa, 1 (1), 8-30 